\title{
Glutathione Conformations and Its Implications for in vivo Magnetic Resonance Spectroscopy
}

\author{
Pravat K. Mandal ${ }^{\mathrm{a}, \mathrm{b}, *}$, Deepika Shukla ${ }^{\mathrm{a}}$, Varan Govind ${ }^{\mathrm{c}}$, Yves Boulard ${ }^{\mathrm{d}}$ and Lars Ersland \\ ${ }^{a}$ Neuroimaging and Neurospectroscopy Laboratory, National Brain Research Center, Gurgaon, India \\ ${ }^{\mathrm{b}}$ The Florey Institute of Neuroscience and Mental Health, Melbourne, VIC, Australia \\ ${ }^{\mathrm{c}}$ Department of Radiology, Miller School of Medicine, University of Miami, FL, USA \\ ${ }^{\mathrm{d}}$ Department of Integrated Biology and Molecular Genetics, Laboratory of Integrated Biology, \\ Saclay Institute of Biology and Technology, CEA-Saclay, Gif-sur-Yvette Cedex, France \\ ${ }^{\mathrm{e}}$ Department of Clinical Engineering, Haukeland University Hospital, Department of Biological \\ and Medical Psychology, University of Bergen, NORMENT - Norwegian Center for Mental Disorders \\ Research, University of Bergen, Bergen, Norway
}

Accepted 10 May 2017

\begin{abstract}
Glutathione (GSH) is a major antioxidant in humans that is involved in the detoxification of reactive radicals and peroxides. The molecular structural conformations of GSH depend on the surrounding micro-environment, and it has been experimentally evaluated using NMR and Raman spectroscopic techniques as well as by molecular dynamics simulation studies. The converging report indicates that GSH exists mainly in two major conformations, i.e., "extended" and "folded". The NMR-derived information on the GSH conformers is essential to obtain optimal acquisition parameters in in vivo MRS experiments targeted for GSH detection. To further investigate the implications of GSH conformers in in vivo MRS studies and their relative proportions in healthy and pathological conditions, a multi-center clinical research study is necessary with a common protocol for GSH detection and quantification.
\end{abstract}

Keywords: Antioxidant, brain, conformation, glutathione, magnetic resonance spectroscopy, molecular dynamics, nuclear magnetic resonance

\section{INTRODUCTION}

Glutathione (GSH) is a tripeptide (L- $\gamma$-glutamylL-cysteinyl-glycine), synthesized in the cytosol from the precursor amino acids glutamate, cysteine, and glycine [1]. GSH is ubiquitous and one of the most abundant metabolites with millimolar $(\mathrm{mM})$

\footnotetext{
*Correspondence to: Dr. Pravat K. Mandal, Professor, Neuroimaging and Neurospectroscopy Laboratory, National Brain Research Center, Gurgaon, India. Tel.: +91 9910318922; E-mails: pravat.mandal@gmail.com; pravat@nbrc.ac.in.
}

intra-cellular concentration. It is the major lowmolecular weight thiol compound in animal cells. Presence of thiol group in GSH makes it susceptible to oxidation [2] as well as possible source of conformational changes due to changes in its surrounding microenvironment. Published reports of molecular dynamics (MD) studies [3-6] as well as studies utilizing state-of-the-art Raman spectroscopy $[7,8]$ and nuclear magnetic resonance (NMR) $[9,10]$ techniques on GSH in various experimental conditions (solvent, $\mathrm{pH}$, temperature, etc.), provide a clear insight into possible GSH conformations (Fig. 1). 
Molecular dynamics is a computer simulation method for studying movements of atoms and molecules, in which an initially created molecular structure is first energy minimized and subsequently the coordinates of structures with lower energy states as conformers are obtained by allowing the atoms and molecules to interact with each other for a fixed period of time. MD simulations over $20 \mathrm{~ns}$ using Gromacs all-atom force field, coupled with cluster analyses of the trajectories, have been applied to examine the distribution proportion of GSH conformations in aqueous solutions as a function of $\mathrm{pH}$ [3]. The results derived from MD simulations show that GSH is very flexible and does not adopt a strongly preferred conformation at any $\mathrm{pH}$ [3]. Calculations in another MD study with GSH [4] were carried out using a modified TINKER 4.2 molecular modeling package at $\mathrm{T}=298 \mathrm{~K}$ and 1 atmospheric pressure. This study also indicated that GSH is highly flexible in an aqueous solution with transitions occurring between the extended and folded conformations [4]. In contrast to classical MD with the motion of interactive atoms, recent advancements in conformal studies are adopting quantum molecular dynamics (QMD). QMD is based on the existence of chemical bonds as a result of electron interactions and can describe the formation and breaking of chemical bonds, which cannot be accomplished using classical molecular dynamics. QMD includes elemental interactions between atoms as well as electrons. QMD simulation methods work on "first principle" based only on the laws of quantum mechanics and thus do not require any prior knowledge on inter-atomic interactions. Behavioral modeling of molecules in QMD is described in terms of density functional theory (DFT), which is presently the most successful approach to compute the electronic structure of matter [5]. The DFT approach predicts a great variety of molecular properties that include molecular structures, vibrational frequencies, atomization energies, ionization energies, electric and magnetic properties, and reaction paths. A recent report on DFT investigation of GSH has also confirmed the extended and folded conformers of GSH reported in an MD study [6].

Conformations of GSH in aqueous solutions have been investigated using Raman spectroscopic methods that rely on vibrational and/or rotational frequency differences. Raman spectroscopy provides detailed information on vibrational frequencies specific to chemical bonds as well as on molecular symmetry, thereby giving a unique fingerprint of the molecule of interest. Since Raman spectroscopy is a non-destructive technique, it is useful for the analysis of chemical structures and their molecular conformations [7]. The amide III band components, between $1308 \mathrm{~cm}^{-1}$ and $1288 \mathrm{~cm}^{-1}$, show three distinct distribution of conformations of GSH ( $\mathrm{P}_{\mathrm{II}}, \alpha_{\mathrm{R}}$ and $\beta$ at $0.5 \mathrm{M}$ GSH in $\mathrm{H}_{2} \mathrm{O}$, pH 7 [8], and these conforma-
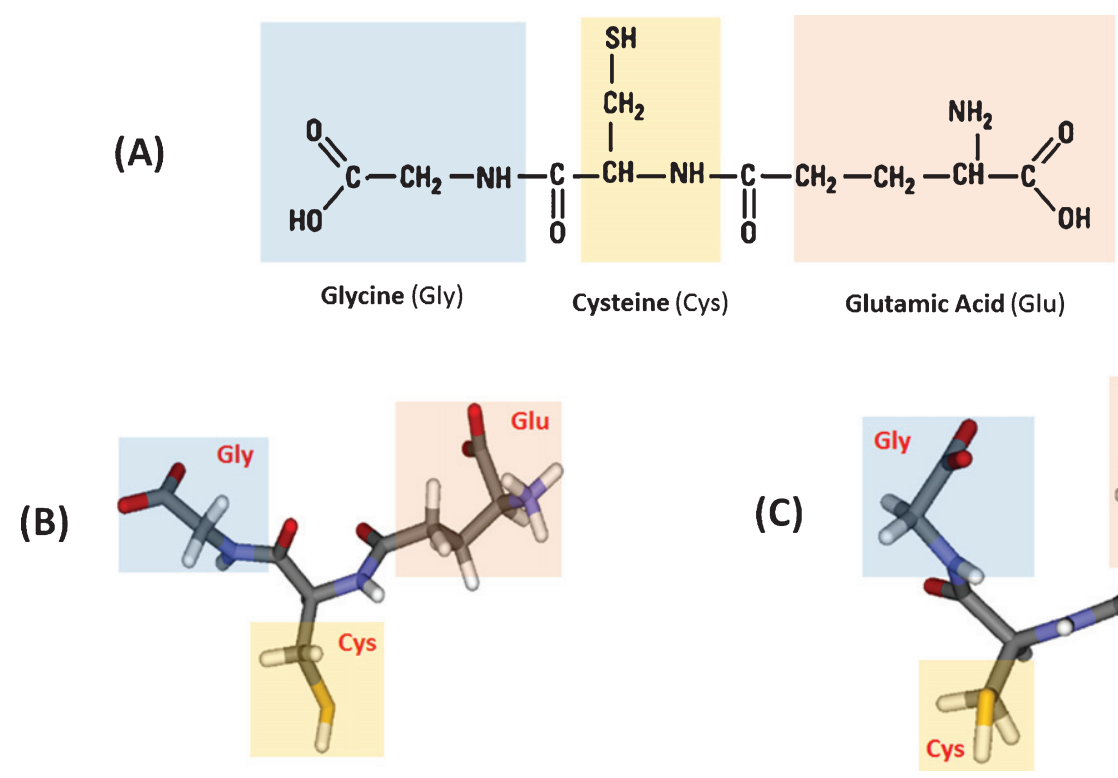

(C)

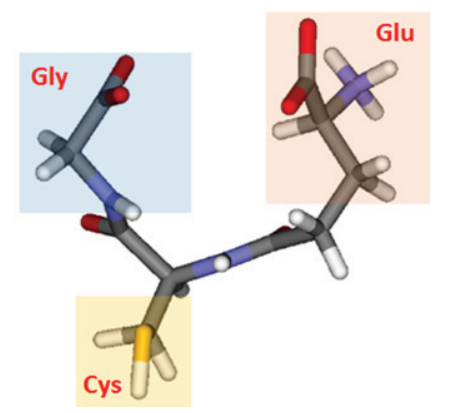

Fig. 1. Glutathione and its structural conformations. (A) Molecular structure of glutathione, (B) the extended, and (C) the folded conformations (modified from [4]; copyright permission obtained from the publisher). 
tions have been used by numerous experimental and theoretical studies. The Raman spectroscopic bands at $1305 \mathrm{~cm}^{-1,} 1298 \mathrm{~cm}^{-1}$ and $1288 \mathrm{~cm}^{-1}$ correspond to the $\mathrm{P}_{\mathrm{II}}, \alpha_{\mathrm{R}}$, and $\beta$ conformations, respectively, of GSH in aqueous medium [8]. The relative population of $\beta, \mathrm{P}_{\mathrm{II}}$, and $\alpha_{\mathrm{R}}$ conformations of GSH is reported as $60 \%, 25 \%$, and $15 \%$, respectively, based on calculations of the amide III regions in Raman spectra of GSH [8].

NMR spectroscopy provides information on the conformational changes of peptides and proteins through measurement of chemical shifts of molecular groups and coupling constants of the chemical bonds in them. Conformational analysis of a compound at a given experimental condition (e.g., pH, temperature and solvent) is accomplished using NMR spectroscopy to determine the relative populations of conformers in solution. Proton NMR chemical shift values of the molecular groups of GSH were determined without ambiguity using one dimensional and/or two-dimensional NMR studies performed at high magnetic fields (400-700 MHz) [9-12] in various physiological conditions (e.g., $\mathrm{pH}$, temperature, and solvent).

The (two) published NMR reports of GSH indicate that there are major differences in the way the samples were prepared [11, 12]. One report [11] used a degassed aqueous GSH solution in order to reduce the amount of dissolved oxygen in the solution, thereby minimizing the oxidation of GSH, while another report [12] used a non-degassed GSH aqueous solution in the NMR studies. GSH is susceptible to oxidation due to the presence of a thiol group, and thus it is highly sensitive to external environment. Therefore, the sample preparation method plays a key role in deciding the conformational state of GSH. The two NMR studies $[11,12]$ that have used a degassed or a non-degassed aqueous GSH solution report distinct conformational states of GSH. NMR studies of a non-degassed aqueous GSH solution show the chemical shift of GSH cysteine $\mathrm{H}_{\alpha}$ and $\mathrm{H}_{\beta}$ at 4.56 and $2.95 \mathrm{ppm}$ [12-14]. In contrast, the studies that have used degassed samples report GSH cysteine $\mathrm{H}_{\alpha}$ and $\mathrm{H}_{\beta}$ at 4.40 and $2.80 \mathrm{ppm}[11,15]$. One of the studies reported the chemical shifts of GSH cysteine $\mathrm{H}_{\alpha}$ and $\mathrm{H}_{\beta}$ at 4.37 and $2.77 \mathrm{ppm}$ in aqueous solution using DMSO as an internal reference [10]. The chemical shift values [10] of the cysteine $\mathrm{H}_{\alpha} / \mathrm{H}_{\beta}$ are close to the values reported in studies that used degassed GSH aqueous solutions [11, 15]. A combined MD and NMR study reveals that GSH in DMSO solution remains in the stable folded conformation, whereas in aqueous solution it is highly flexible with transitions between extended and folded conformations [4]. The stability of the folded GSH form has been confirmed by a recent advanced DFT calculations report [6].

Based on these combined studies [4, 6], it can be inferred that chemical shifts of cysteine $\mathrm{H}_{\alpha}$ and $\mathrm{H}_{\beta}$ of GSH at 4.40 and $2.80 \mathrm{ppm}$, respectively, in degassed aqueous solution is similar to the one obtained in DMSO solution, are indicative of the stable folded GSH conformation. We hypothesize that reduced glutathione in aqueous solution has two conformations as indicated by MD studies: the extended conformation is characterized by the chemical shifts of cysteine $\mathrm{H}_{\alpha}$ and $\mathrm{H}_{\beta}$ at 4.56 and $2.95 \mathrm{ppm}$, respectively, and the folded conformation is characterized by the chemical shifts of $\mathrm{H}_{\alpha}$ and $\mathrm{H}_{\beta}$ at 4.40 and $2.80 \mathrm{ppm}$, respectively. However, there is need to perform a thorough and well-controlled experimental study to understand the reasons behind the changes observed in the chemical shifts of cysteine hydrogens of GSH and to ascertain if this is to do with the change in its conformations or other factors.

Glutathione is a free radical scavenger, which plays a key role in maintaining the oxidative and redox balance in human cells and exists in both the reduced (GSH) and oxidized (GSSG) forms. GSH can be converted to GSSG by the enzyme glutathione peroxidase, and this can be reconverted to GSH by glutathione reductase [16]. Measurements of GSH, GSSG and their enzyme catalyzed reactions are thus important for evaluating the redox and antioxidant status [16]. GSH serves as a marker of oxidative stress that is an important factor in Alzheimer's disease (AD) [17]. GSH in the brain is reported to be $1.18 \pm 0.09 \mathrm{mM}$ from the parietal cortex of autopsy brain [18], which decreases with age and ageassociated disorders [19]. A clinical study indicated the link between oxidative damage, mild cognitive impairment (MCI) and $\mathrm{AD}$ using the plasma levels of GSH and GSH/GSSG ratio of 34 subjects with MCI, 45 subjects with AD, and 28 age-matched control subjects [20]. The results showed a significant decrease in GSH levels and GSH/GSSG ratios in AD and MCI patients compared to age matched control subjects [20]. The depletion of GSH was specific to AD patients compared to patients with other neurodegenerative disorders such as Parkinson's disease and dementia with Lewy body disease [21]. This clinical study reported that the mean GSH levels in the cingulate cortex brain region of $\mathrm{AD}$ patients were 
decreased (49\%) compared to age matched control subjects [21]. These disease specific studies [20,21] stress the importance of quantitation of GSH in the brain regions patients with different pathological conditions (e.g., $\mathrm{AD}$ and $\mathrm{MCI}$ ), which may serve as an early surrogate marker of diseases.

\section{DISCUSSION}

It has been established that the tripeptide structure of GSH is quite sensitive to its surrounding environment (e.g., pH, temperature, and solvent). Molecular dynamics studies have shown that the conformation of GSH is very flexible in aqueous solution by converting it from the extended form to the folded form [4]. Furthermore, NMR and Raman spectroscopic studies of GSH clearly support the existence of various conformers of it. The results of these studies allude to the presence or absence of anaerobic or aerobic environment indeed influence the antioxidant potential of GSH by modulating the conformational changes of GSH. At present, it is not known which of the two GSH conformations (i.e., extended or folded) is present in the human brain. It is pertinent to know the proportion of the two GSH conformations in healthy brain and in pathological conditions. Answers to these questions will have major implications in the way GSH levels are detected and quantitated using in vivo MRS methods.

It is prudent to report that the accuracy of the NMR spectral parameters (chemical shifts and coupling constants) of glutathione can be limited for its molecular groups with complex and overlapping spectral patterns. Furthermore, the values for these parameters may be altered in in vivo conditions as a result of local physiological and cellular environment [22]. Detection of GSH in human brain in vivo is commonly accomplished by ${ }^{1} \mathrm{H}$ magnetic resonance spectroscopy (MRS) using spectrally-selective editing schemes that employ J-couplings of cysteinal hydrogens. We hypothesize that the proton NMR spectral peak positions of cysteine $\mathrm{H}_{\alpha}$ and $\mathrm{H}_{\beta}$ are highly associated with the conformational states of GSH. The QMD study of GSH [6] has not got sufficient attention, and further studies with in vivo physiological conditions are necessary to understand possible conformations of GSH in the brain. A clear understanding for the conformations of GSH in the brain will help us to come up with optimal experimental data acquisition parameters for MRS studies performed in vivo for selectively detecting GSH.
An important first step is to do thorough in vitro NMR experiments of GSH in different physiological conditions that will clarify some of the unanswered questions. Towards this, we plan to acquire proton MRS data at high magnetic fields (e.g., >11 T) using phantoms containing GSH and/or GSSG at physiological and non-physiological (or pathological) conditions as well as ex vivo intact animal or human brain tissue samples. The high magnetic field MRS data will allow us to measure the chemical shifts and coupling constants of the hydrogens in the extended and folded GSH conformations at much higher accuracy than at 3T clinical MRI scanner.

Previous task force reported eight neurochemical (not GSH) with their specific chemical shifts and in vivo concentration range. Subsequently, a multicenter in vivo research project with a common data acquisition protocol will help us to unravel the associations between the conformations of GSH in vivo and the observed spectral patterns in healthy controls and pathological conditions.

\section{ACKNOWLEDGMENTS}

Dr. Pravat K. Mandal (Principal Investigator) thanks the Department of Biotechnology, Government of India for funding this project (Grant No BT/PR7361/MED/30/953/2013). Dr. Govind received support from the National Institutes of Health (NIH) R01 grants NS060874 and NS094043.

Authors' disclosures available online (http://j-alz. com/manuscript-disclosures/17-0350r1).

\section{REFERENCES}

[1] Chakravarthi S, Jessop CE, Bulleid NJ (2006) The role of glutathione in disulphide bond formation and endoplasmicreticulum-generated oxidative stress. EMBO Rep 7, 271275.

[2] Ying J, Clavreul N, Sethuraman M, Adachi T, Cohen RA (2007) Thiol oxidation in signaling and response to stress: Detection and quantification of physiological and pathophysiological thiol modifications. Free Radic Biol Med 43, 1099-1108.

[3] Lampela O, Juffer AH, Rauk A (2003) Conformational analysis of glutathione in aqueous solution with molecular dynamics. J Phys Chem A 107, 9208-9220.

[4] Zhang R, Wu W, Luo S (2011) Different behaviors of glutathione in aqueous and DMSO solutions: Molecular dynamics simulation and NMR experimental study. $J$ Solution Chem 40, 1784-1795.

[5] Seifert G (2008) Quantum molecular dynamics: The physics of explosive chemistry. Nat Physics 4, 12-13.

[6] Singh G, Dogra SD, Kaur S, Tripathi SK, Prakash S, Rai B, Saini GS (2015) Structure and vibrations of glutathione 
studied by vibrational spectroscopy and density functional theory. Spectrochim Acta A Mol Biomol Spectrosc 149, 505-515.

[7] Wood S, Hollis JR, Kim JS (2017) Raman spectroscopy as an advanced structural nanoprobe for conjugated molecular semiconductors. J Phys D Appl Phys 50, DOI: 10.1088/13 61-6463/50/7/073001

[8] Glusic M, Ropret P, Vogel-Mikus K, Grdadolnik J (2013) The binding sites of cadmium to a reduced form of glutathione. Acta Chim Slov 60, 61-69.

[9] York MJ, Beilharz GR, Kuchel PW (1987) Conformation of reduced glutathione in aqueous solution by $1 \mathrm{H}$ and $13 \mathrm{C}$ n.m.r. Int J Pept Protein Res 29, 638-646.

[10] Duddeck H, Dietrich W, Tóth G (1998) Structure elucidation by modern NMR: A workbook, Steinkopff, Springer, New York, pp. 76-78.

[11] Delalande O, Desvaux H, Godat E, Valleix A, Junot C, Labarre J, Boulard Y (2010) Cadmium-glutathione solution structures provide new insights into heavy metal detoxification. FEBS J 277, 5086-5096.

[12] Govindaraju V, Young K, Maudsley AA (2000) Proton NMR chemical shifts and coupling constants for brain metabolites. NMR Biomed 13, 129-153.

[13] Kato N, Nakamura M, Uchiyama T (1999) 1H NMR studies of the reactions of copper(I) and copper(II) with D-penicillamine and glutathione. J Inorg Biochem 75, 117-121.

[14] Kennett EC, Bubb WA, Bansal P, Alewood P, Kuchel PW (2005) NMR studies of exchange between intra- and extracellular glutathione in human erythrocytes. Redox Rep 10, 83-90.
[15] Rey NA, Howarth OW, Pereira-Maia EC (2004) Equilibrium characterization of the As(III)-cysteine and the As(III)glutathione systems in aqueous solution. J Inorg Biochem 98, 1151-1159.

[16] Yao JK, Leonard S, Reddy R (2006) Altered glutathione redox state in schizophrenia. Dis Markers 22, 83-93.

[17] Smith MA, Nunomura A, Zhu X, Takeda A, Perry G (2000) Metabolic, metallic, and mitotic sources of oxidative stress in Alzheimer disease. Antioxid Redox Signal 2, 413-420.

[18] Franz Hefti F, Weiner WJ (1988) Progress in Parkinson Research. Plenum Press, New York and London.

[19] Liu H, Wang H, Shenvi S, Hagen TM, Liu RM (2004) Glutathione metabolism during aging and in Alzheimer disease. Ann N Y Acad Sci 1019, 346-349.

[20] Bermejo P, Martin-Aragon S, Benedi J, Susin C, Felici E, Gil P, Ribera JM, Villar AM (2008) Peripheral levels of glutathione and protein oxidation as markers in the development of Alzheimer's disease from Mild Cognitive Impairment. Free Radic Res 42, 162-170.

[21] Gu M, Owen AD, Toffa SE, Cooper JM, Dexter DT, Jenner P, Marsden CD, Schapira AH (1998) Mitochondrial function, GSH and iron in neurodegeneration and Lewy body diseases. J Neurol Sci 158, 24-29.

[22] Govind V, Young K, Maudsley AA (2015) Corrigendum: Proton NMR chemical shifts and coupling constants for brain metabolites. Govindaraju V, Young K, Maudsley AA, NMR Biomed. 2000; 13:129-153. NMR Biomed 28, 923-924. 